\title{
On Certain Bounds for Edge Metric Dimension of Zero-Divisor Graphs Associated with Rings
}

\author{
Hafiz Muahmmad Afzal Siddiqui $\left(D,{ }^{1}\right.$ Ammar Mujahid, ${ }^{1}$ Muhammad Ahsan Binyamin, ${ }^{2}$ \\ and Muhammad Faisal Nadeem (iD) ${ }^{1}$ \\ ${ }^{1}$ COMSATS University Islamabad, Lahore-Campus, Department of Mathematics, Defence Road Lahore, Islamabad, Pakistan \\ ${ }^{2}$ Government College University Faisalabad, Department of Mathematics, Faisalabad, Pakistan
}

Correspondence should be addressed to Hafiz Muahmmad Afzal Siddiqui; hmasiddiqui@gmail.com

Received 17 August 2021; Accepted 25 November 2021; Published 30 December 2021

Academic Editor: Amin Jajarmi

Copyright (C) 2021 Hafiz Muahmmad Afzal Siddiqui et al. This is an open access article distributed under the Creative Commons Attribution License, which permits unrestricted use, distribution, and reproduction in any medium, provided the original work is properly cited.

\begin{abstract}
Given a finite commutative unital ring $\mathcal{S}$ having some non-zero elements $x, y$ such that $x \cdot y=0$, the elements of $\mathcal{S}$ that possess such property are called the zero divisors, denoted by $\mathscr{Z}(S)$. We can associate a graph to $\delta$ with the help of zero-divisor set $\mathscr{Z}(S)$, denoted by $\zeta(S)$ (called the zero-divisor graph), to study the algebraic properties of the ring $\mathcal{S}$. In this research work, we aim to produce some general bounds for the edge version of metric dimension regarding zero-divisor graphs of $\mathcal{S}$. To do so, we will discuss the zero-divisor graphs for the ring of integers $\mathbb{Z}_{m}$ modulo $m$, some quotient polynomial rings, and the ring of Gaussian integers $\mathbb{Z}_{m}[i]$ modulo $m$. Then, we prove the general result for the bounds of edge metric dimension of zero-divisor graphs in terms of maximum degree and diameter of $\zeta(S)$. In the end, we provide the commutative rings with the same metric dimension, edge metric dimension, and upper dimension.
\end{abstract}

\section{Introduction}

The connection between two mainstream mathematics fields algebra and graph theory was first proposed by Beck [1]. Initially, he introduced the concept of zero-divisor graph associated to a commutative ring $\mathcal{S}$, where he considered every element of a ring $\mathcal{S}$ as the vertices of zero-divisor graph and those two distinct vertices $r$ and $s$ are connected for which $r \cdot s=0$. Observe that in this case, the 0 vertex is connected to every other vertex. In literature, this type of zero-divisor graph is denoted by $\zeta^{\circ}(S)$. In this work of Beck, his main idea was to present the coloring of a commutative ring. This investigation of coloring of a commutative ring was further analyzed by Anderson and Naseer in [2]. Also, they provided a counter example to Beck who conjectured that clique number $c l(S)$ and chromatic number $\chi(S)$ of a ring $\mathcal{S}$ are the same by showing that for a finite local ring $\mathcal{S}$, $c l(S)=5$ and $\chi(S)=6$.

The zero-divisor graphs by means of zero divisors $\mathscr{E}(S)$ of a unital commutative ring $\mathcal{S}$ were studied by Anderson and Livingston in [3], and we will denote this type of zerodivisor graphs by $\zeta(S)$. This definition of zero-divisor graph is slightly different from Beck's definition of zero-divisor graph associated to a commutative ring. Observe that, in this case, the element 0 is not considered as the vertex of zerodivisor graph, and so $\zeta(S)$ is a subgraph of $\zeta^{\circ}(S)$. Anderson and Livingston presented the interplay between the ring theoretic properties of $\delta$ and the graph theoretic properties of $\zeta(S)$; furthermore, this research provides some fundamental results related to zero-divisor graph $\zeta(S)$.

This concept of zero-divisor graphs associated to a unital commutative ring was then extended by means of noncommutative rings by Redmond [4]. He introduced various ways to define the zero-divisor graph associated to a noncommutative ring, which includes both directed and undirected graphs. This work was then continued by Redmond [5] by means of zero-divisor graph of a commutative ring to an ideal-based zero-divisor graph of a commutative ring, where he thought of generalizing this approach by replacing elements whose product is zero with elements whose 
product lies in some ideal $\mathscr{I}$ of $\mathcal{S}$. An ideal-based zerodivisor graph with the vertex set $\{r \in \mathcal{S} / \mathscr{I}: r s \in \mathscr{I}$ for some $s \in \mathcal{S} / \mathscr{I}\}$, where two different vertices $r$ and $s$ are connected if and only if their product lies in the ideal $\mathscr{F}$, is denoted by $\zeta_{\mathscr{F}}(S)$. Since then, many authors have been working on this and defined the graphs such as unit graphs, zero-divisor graphs of equivalence classes, total graphs, ideal-based zero-divisor graphs, Jacobson graphs, and so on (see, for example, [5-9]). For graph theory, we refer the readers to $[10,11]$, and for basic definitions in ring theory, we refer the readers to $[12,13]$.

Redmond [14] discussed all the commutative rings with unity (up to isomorphism) which produces the zero-divisor graphs on $m=6,7, \ldots, 14$ vertices. Moreover, an algorithm is provided to determine all commutative rings (up to isomorphism) with unity. Redmond and Szabo in [15] discussed the zero-divisor graphs of commutative rings with different metrics and upper dimensions. Ali provided a survey on antiregular graphs in [16]. The graph associated to a commutative ring is surprisingly the best demonstration of the properties of the zero-divisor set of a ring. This graph allows and helps us to figure out the algebraic properties of rings using graph theoretic approaches. The authors in [3] discussed some interesting properties of $\zeta(S)$. We use the way adopted by Anderson and Livingston in [3], by considering the set of non-zero zero divisors $\mathscr{Z}(S)$ as the vertex set for $\zeta(S)$.

Throughout the paper, $\delta$ is assumed to be a finite unital commutative ring, unless otherwise stated. $\mathscr{Z}(S)$ is the set of non-zero zero divisors as discussed above. A ring $\mathcal{S}$ is called local if it has a unique maximal ideal. The annihilator ann $(r)$ of an element $r \in \mathcal{S}$ is defined as $\{s \in \mathcal{S}: r s=0\}$. An element $r \in \mathcal{S}$ is called nilpotent if $r^{m}=0$ for some positive integer $m$. A ring $\mathcal{S}$ is called reduced if it has no non-zero nilpotent elements. $\mathbb{Z}_{m}=\{0,1,2, \ldots, m-1\}$ is the ring of integers modulo $m$, $\mathbb{Z}_{m}[i]=\left\{x+i \cdot y: x, y \in \mathbb{Z}_{m}\right.$ and $\left.i^{2}=-1\right\}$ is the ring of gaussian integers modulo $m$ with respect to the usual complex addition and multiplication, and $\mathbb{K}_{m}$ stands for a finite field. The graph associated to the ring of Gaussian integers modulo $m$ was introduced by Osba et al. in [17], and the zero-divisor graph of the ring of gaussian integers $\mathbb{Z}_{m}[i]$ is denoted by $\zeta\left(\mathbb{Z}_{m}[i]\right)$

Kelenc et al. introduced the concept of edge version of metric dimension in [18]. Consider a graph $G$ having arbitrary vertices $r$ and $s$ and an edge $t=r s$; the mapping $d: V(G) \cup E(G) \longrightarrow\{0,1,2,3, \ldots n-1\} \quad$ defined by $d(w, t)=\min \{d(r, t), d(s, t)\}$ is the distance between $w$ and $t$. Any two edges $t_{1}$ and $t_{2}$ of $G$ are distinguished by a vertex $w$ of $G$ only if $d\left(w, t_{1}\right) \neq d\left(w, t_{2}\right)$. Any subset $S$ of vertex set of $G$ is called an edge resolving set for $G$ if every pair of edges of $G$ is distinguished by some vertex set of $S$. The cardinality of the smallest edge resolving set of $G$ is called the edge metric dimension of $G$ and is denoted by $\operatorname{dim}_{E}(G)$. An edge resolving set $S$ of a connected graph $G$ uniquely codes all the edges of $G$.

\section{Preliminaries}

A graph $G(V, E)$ consists of a vertex set $V$ and an edge set $E$, and the number $|V|$ denotes the order of $G$, whereas the number $|E|$ denotes the size of $G$. An edge $t \in E(G)$ relates to a pair of distinct vertices, say $r$ and $s$, written as $t=r s$. An alternating arrangement among vertices and edges is known as a walk. If we traverse a graph $G$ such that no vertex and edge is repeated, then it is known as a path. If the initial vertex and the terminal vertex in a path are the same, then it is known as a cycle. The distance between two distinct vertices $r$ and $s$ is the number of edges in the smallest path among them, and it is denoted by $d(r, s)$, and if there does not exist a path among them, we define $d(r, s)$ to be infinite. If $d(r, s)=1$, then $r$ and $s$ are said to be neighboring vertices. The neighborhood set of a vertex $r \in V$ is $N(r)=\{s \in V: r s \in E\} ; \quad$ furthermore, $\quad N[r]=N(r) \cup\{r\}$ denotes the set of closed neighborhood of $r \in V$. The number $|N(r)|$ is the degree of the vertex $r \in V$ which is denoted by $\operatorname{deg}(r)$ or simply $d(r)$. If for every $r \in V, d(r)=c$ for some fix $c \in \mathbb{Z}^{+}$, then the graph is said to be regular graph. The number $d(u, t)=\min \{d(u, r), d(u, s)\}$ represents the distance between vertex $u$ and an edge $t=r s$. The length of the longest path is the diameter of the graph which is denoted by $\operatorname{diam}(G) . \quad$ Mathematically, $\quad \operatorname{diam}(G)=\sup \{d(r, s)$ $: r$ and $s$ are distinct vertices in $G$ \}.

Any subset $H$ of vertices together with any subset of edges containing those vertices is a subgraph of a graph $G$; mathematically, we write $H \subset G$. The number of edges in the smallest cycle subgraph in a graph $G$ is called the girth of graph, denoted by $\operatorname{gr}(G)$. The maximal complete subgraph of a graph $G$ is called a clique which is denoted by $K$ and $|K|=\omega(G)$ is called the clique number. If there is an edge among every pair of vertices in a graph, then it is said to be complete graph which is denoted by $K_{m}$, where $m$ is the number of vertices. If the vertices of a graph can be partitioned into two disjoint sets, say $X$ and $Y$ such that each vertex of $X$ is adjacent to each vertex in $Y$, then the graph is said to be complete bipartite graph, and it is usually denoted by $K_{|X|,|Y|}$ or simply $K_{m, n}$ when $|X|=m$ and $|Y|=n$. A cut vertex is a vertex that when removed from a connected graph creates two or more components of the graph.

Kelenc et al. in [18] discussed the edge metric dimension of the path graph, complete graph, and complete bipartite graph. Since both the metric dimension and the edge metric dimension are closely related, it is feasible to find out graphs for which the metric dimension and the edge metric dimension are the same, as well as for some other graphs $G$ for which $\operatorname{dim}(G)<\operatorname{dim}_{E}(G)$ or $\operatorname{dim}_{E}(G)<\operatorname{dim}(G)$. In fact, Kelenc et al. were interested in exploring the comparison between the values of $\operatorname{dim}(G)$ and $\operatorname{dim}_{E}(G)$.

The edge metric dimension of the path graph $P_{m}$, cycle graph $C_{m}$, and the complete graph $K_{m}$ is given in the following results.

Theorem 1 (see [18], Remark 1]). For any integer $m \geq 2$, $\operatorname{dim}_{E}\left(P_{m}\right)=\operatorname{dim}\left(P_{m}\right)=1, \operatorname{dim}_{E}\left(C_{m}\right)=\operatorname{dim}\left(C_{m}\right)=2$, and $\operatorname{dim}_{E}\left(K_{m}\right)=\operatorname{dim}\left(K_{m}\right)=m-1$. Moreover, $\operatorname{dim}_{E}(G)=1$ if and only if $G \cong P_{m}$.

Next, it is shown that for a complete bipartite graph $K_{r, s}$ different from $K_{1,1}$, the edge metric dimension is $r+s-2$. 
Theorem 2 (see [18], Remark 2]). For any complete bipartite graph $K_{r, s}$ such that $r \geq 1$ and $s \geq 2$, $\operatorname{dim}_{E}\left(K_{r, s}\right)=\operatorname{dim}\left(K_{r, s}\right)=r+s-2$.

\section{Edge Metric Dimension of Graphs Associated with Rings}

For a graph $G$ of single vertex, the edge metric dimension is assumed to be zero and for an empty graph, the edge metric dimension is undefined. So, we begin our discussion with the following observation.

Theorem 3. Let $\mathcal{S}$ be a finite commutative ring with unity. Then,

(i) $\operatorname{dim}_{E}(\zeta(\mathcal{S}))$ is finite iff $\mathcal{S}$ is finite.

(ii) $\operatorname{dim}_{E}(\zeta(\mathcal{S}))$ is undefined iff $\mathcal{S}$ is an integral domain.

Proof

(i) Suppose that $\operatorname{dim}_{E}(\zeta(\delta))$ is finite; then, there exists a minimal edge metric basis for $(\zeta(\delta)$ ), say $\left\{v_{1}, v_{2}, \ldots, v_{t}\right\}$. By ([3], Theorem 2.3) $\operatorname{diam}(\zeta(\mathcal{S})) \leq 3$. So, $d(r, e)=0,1,2$ or 3 for every $r \in V(\zeta(\mathcal{S}))$ and $e \in E(\zeta(\mathcal{S}))$. Hence, $|\mathscr{Z}(\mathcal{S})| \leq 4^{t}$, which implies that $\mathscr{Z}(\mathcal{S})$ is finite, and hence $\mathcal{S}$ is finite. Conversely, given that $\mathcal{S}$ is finite, then $|\mathscr{Z}(\mathcal{S})|$ is finite, since $\mathscr{Z}(\mathcal{S})$ is contained in $\mathcal{S}$. So, $\operatorname{dim}_{E}(\zeta(\delta))$ is finite.

(ii) As we know that edge metric dimension of $\zeta(\mathcal{S})$ is undefined whenever $\mathcal{S}$ is an integral domain and vice versa, the assertion follows.

The following result gives the edge metric dimension of the zero-divisor graphs of a ring $\mathcal{S}$ whenever $\zeta(\mathcal{S})$ is isomorphic to $P_{m}$ for some $m$.

Proposition 1. Let $\mathcal{S}$ be a finite commutative ring with unity. Then, $\operatorname{dim}_{E}(\zeta(\mathcal{S}))=1$ if and only if $\mathcal{S}$ is isomorphic to one of the following rings: $\mathbb{Z}_{6}, \mathbb{Z}_{8}, \mathbb{Z}_{9}, \mathbb{Z}_{2} \times \mathbb{Z}_{2}, \mathbb{Z}_{3}[r] /\left(r^{2}\right)$, $\mathbb{Z}_{2}[r] /\left(r^{3}\right)$, or $\mathbb{Z}_{4}[r] /\left(2 r, r^{2}-2\right)$.

Proof. Suppose that $\operatorname{dim}_{E}(\zeta(\delta))=1$; then, by Theorem 1, paths are the only graphs whose edge metric dimension is 1 , so $\zeta(\mathcal{S}) \cong P_{m}$. Since $|\mathscr{Z}(\mathcal{S})|$ is not more than 3 whenever $\zeta(\mathcal{S})$ is a path graph by ([19], Lemma 2.6), $\zeta(\mathcal{S})$ is either $P_{2}$ or $P_{3}$.

Case I. If $\zeta(\mathcal{S}) \cong P_{2}$, then $\mathscr{Z}(\mathcal{S})=\{a, b\}$ such that $a b=0$. The rings which satisfy this property are $\mathbb{Z}_{9}$, $\mathbb{Z}_{2} \times \mathbb{Z}_{2}$, and $\mathbb{Z}_{3}[r] /\left(r^{2}\right)$.

Case II. If $\zeta(\mathcal{S}) \cong P_{3}$, then $\mathscr{Z}(\mathcal{S})=\{a, b, c\}$, such that $a b=0$ and $b c=0$. The rings which satisfy this property are $\mathbb{Z}_{6}, \mathbb{Z}_{8}, \mathbb{Z}_{2}[r] /\left(r^{2}\right)$, and $\mathbb{Z}_{4}[r] /\left(2 r, r^{2}-2\right)$ [14].

Conversely, the zero-divisor graphs of above given rings are either $P_{2}$ or $P_{3}$ [14]. Also, the zero-divisor relation is not transitive for these rings. Hence, by Theorem 1, $\operatorname{dim}_{E}(\zeta(\mathcal{S}))=1$.
Proposition 2. Let $\mathcal{S}$ be a finite commutative ring with unity and $\zeta(\mathcal{S}) \cong C_{m}$. Then, $\mathcal{S}$ is isomorphic to one of these rings:

$$
\begin{aligned}
\text { (a) } & \mathbb{Z}_{3} \times \mathbb{Z}_{3} \text {. } \\
\text { (b) } & \mathbb{K}_{4}[r] /\left(r^{2}\right), \quad \mathbb{Z}_{4}[r] /\left(r^{2}+r+1\right), \quad \mathbb{Z}_{4}[r] /(2, r)^{2}, \\
& \mathbb{Z}_{2}[r, s] /(r, s)^{2} .
\end{aligned}
$$

Proof. Given that $\mathcal{S}$ is a commutative ring with unity and $\zeta(\mathcal{S})$ is a cycle graph, then by ([3], Theorem 2.4) the length of the cycle graph cannot exceed 4 . We have shown the zerodivisor graphs of the above given rings in Figure 1.

Corollary 1. Let $\mathcal{S}$ be a finite commutative ring with unity. Then, $\operatorname{dim}_{E}(\zeta(\mathcal{S}))=2$ if $\mathcal{S}$ is isomorphic to one of the following rings: $\mathbb{Z}_{3} \times \mathbb{Z}_{3}, \quad \mathbb{K}_{4}[r] /\left(r^{2}\right), \mathbb{Z}_{4}[r] /\left(r^{2}+r+1\right)$, $\mathbb{Z}_{4}[r] /(2, r)^{2}, \mathbb{Z}_{2}[r, s] /(r, s)^{2}$.

Theorem 4. Let $\mathcal{S}$ be a finite commutative ring with unity such that each $r \in \mathscr{Z}(\mathcal{S})$ is nilpotent.

(1) If $|\mathscr{Z}(\mathcal{S})| \geq 3$ and $\mathscr{Z}(\mathcal{S})^{2}=\{0\}$, then $\operatorname{dim}_{E}(\zeta(\mathcal{S}))=|\mathscr{Z}(\mathcal{S})|-1$.

(2) If $|\mathscr{Z}(\mathcal{S})| \geq 3$ and $\mathscr{Z}(\mathcal{S})^{2} \neq\{0\}$, then $\operatorname{dim}_{E}(\zeta(\mathcal{S}))=$ $|\mathscr{Z}(\mathcal{S})|-2$.

Proof

(1) Given that $|\mathscr{Z}(\mathcal{S})| \geq 3$ and $\mathscr{Z}(\mathcal{S})^{2} \neq\{0\}$, then $r s=0$ for all $r, s \in \mathscr{Z}(\mathcal{S})$, and so by ([5], Theorem 2.8), $\zeta(\mathcal{S})$ is complete graph. Hence, $\operatorname{dim}_{E}(\zeta(\mathcal{S}))=|\mathscr{Z}(\mathcal{S})|-1$ by Theorem 1 .

(2) Given that $|\mathscr{Z}(\mathcal{S})| \geq 3$ and $\mathscr{Z}(\mathcal{S})^{2} \neq\{0\}$, then there exist some $r \in \mathscr{Z}(\mathcal{S})$ such that $r^{2} \neq 0$ which implies that there exists $s \in \mathscr{Z}(\mathcal{S})$ such that $d(r, s) \geq 2$. Hence, $\mathscr{Z}(\delta) /\{t, s\}$ is an edge metric generator for any vertex $t$ adjacent to $r$; therefore, $\operatorname{dim}_{E}(\zeta(\delta)) \leq|\mathscr{Z}(\mathcal{\delta})|-2$.

By ([20], Proposition 1), if $\mathcal{S}$ is a commutative ring with unity, the cut vertex of $\zeta(\mathcal{S})$ is in the center of $\zeta(\delta)$. By ([20], Lemma 4), it is shown that if $\mathcal{S}$ is a finite commutative ring with unity, then $\zeta(\delta)$ has a cut vertex of degree 1 if and only if either there is some $r \in \mathcal{S}$ such that $|\operatorname{ann}(r)|=2$ or $\mathcal{S}$ is isomorphic to $\mathbb{Z}_{9}$ or $\mathbb{Z}_{3}[r] /\left(r^{2}\right)$. The next theorem provides the edge metric dimension of $\zeta(\mathcal{S})$ when $\zeta(\mathcal{S})$ has a cut vertex but not degree 1 vertex.

Theorem 5. Let $\delta$ be a finite commutative ring with unity such that $|\mathscr{Z}(\mathcal{S})| \geq 3$. If $\zeta(\mathcal{S})$ has a cut vertex but no degree 1 vertex, then $\operatorname{dim}_{E}(\zeta(\mathcal{S}))=5$.

Proof. Let $\mathcal{S}$ be a commutative ring with unity; if $\zeta(\mathcal{S})$ has a cut vertex but no degree one vertex, then by ([20], Theorem $3), \mathcal{S}$ is isomorphic to one of the following rings: 


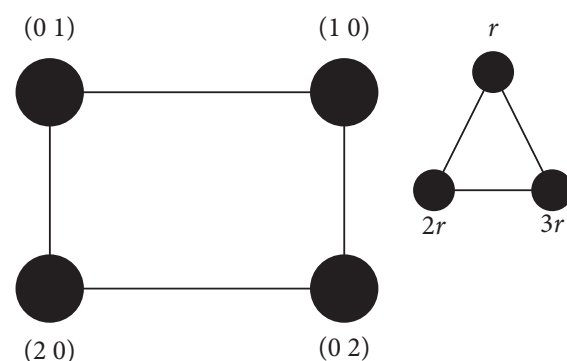

(a)
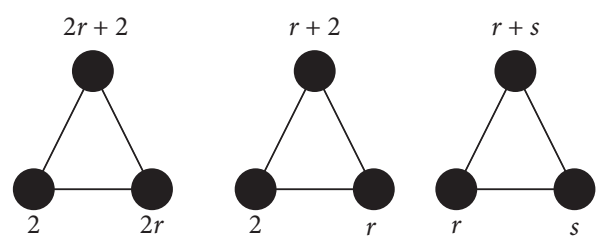

(b)

Figure 1: Zero-divisor graphs of above given rings.

$$
\begin{aligned}
& \frac{\mathbb{Z}_{2}[r, s]}{\left(r^{2}, s^{2}-r s\right)}, \\
& \frac{\mathbb{Z}_{4}[r]}{\left(r^{2}+2 r\right)}, \\
& \frac{\mathbb{Z}_{4}[r, s]}{\left(r^{2}, s^{2}-r s, r s-2,2 r, 2 s\right)}, \\
& \frac{\mathbb{Z}_{8}[r, s]}{\left(2 r, r^{2}+4\right)}, \\
& \frac{\mathbb{Z}_{2}[r, s]}{\left(r^{2}, s^{2}\right)}, \frac{\mathbb{Z}_{4}[r]}{\left(r^{2}\right)}, \\
& \frac{\mathbb{Z}_{4}[r, s]}{\left(r^{2}, s^{2}, r s-2,2 r, 2 s\right)} .
\end{aligned}
$$

The zero-divisor graph associated with first four rings is given in Figure 2(a), and for remaining three rings, the zerodivisor graph is given in Figure 2(b).

The minimum edge metric generator for graphs in Figures 2(a) and 2(b) is given, respectively, by $S_{1}=\left\{v_{1}, v_{2}, v_{3}, v_{4}, v_{6}\right\}$ and $S_{2}=\left\{x_{1}, x_{2}, x_{3}, x_{4}, x_{5}\right\}$. Hence, in both cases, $\operatorname{dim}_{E}(\zeta(\mathcal{S}))=5$.

We next determine the edge metric dimension of $\zeta(\mathcal{S})$, when $\zeta(\delta)$ has exactly one vertex which is adjacent to every other vertex.

Theorem 6. Let $\mathcal{S}$ be a finite commutative ring with unity and $\mathcal{S} \cong \mathbb{Z}_{2} \times \mathbb{K}$ for some finite field $\mathbb{K}$. Then, $\zeta(\mathcal{S}) \cong K_{1,|\mathscr{X}(\mathcal{S})|-1}$ and $\operatorname{dim}_{E}(\zeta(\mathcal{S}))=|\zeta(\mathcal{S})|-2$. Moreover, if $\mathcal{S}$ is a local ring such that $\zeta(\mathcal{S})$ has no cycles, then $\operatorname{dim}_{E}(\zeta(\mathcal{S}))=1$.

Proof. First, we suppose that $\delta$ is a non-local ring and $\mathcal{S} \cong \mathbb{Z}_{2} \times \mathbb{K}$. The set of zero divisors of $\mathcal{S}$ is then $\{(1,0),(0, r): r \in\{1,2, \ldots,|\mathbb{K}|-1\}\} \quad$ such that $(1,0) \cdot(0, r)=0$ for all $r \in\{1,2, \ldots,|\mathbb{K}|-1\}$. Observe that the vertex $u=(1,0)$ is the central vertex and is adjacent to all other $|\mathscr{Z}(\mathcal{S})|-1$ vertices and so $\zeta(\mathcal{S}) \cong K_{1,|\mathscr{E}(\mathcal{S})|-1}$. Hence, by (Theorem 2 ), $\operatorname{dim}_{E}(\zeta(\mathcal{S}))=|\mathscr{Z}(\mathcal{S})|-2$.
On the other hand, if $\mathcal{S}$ is a local ring and has no cycles, then by ([20], Theorem 2.1), $\zeta(\mathcal{S})$ is isomorphic to either $P_{2}$ or $P_{3}$, and hence $\operatorname{dim}_{E}(\zeta(\mathcal{S}))=1$.

Theorem 7. Let $\mathcal{S}$ be a finite commutative ring with unity and $\mathcal{S} \cong \mathbb{K}_{1} \times \mathbb{K}_{2}$, and both $\mathbb{K}_{1}$ and $\mathbb{K}_{2}$ are finite fields with $\left|\mathbb{K}_{1}\right|=m \geq 3, \quad\left|\mathbb{K}_{2}\right|=n \geq 3 . \quad$ Then, $\operatorname{dim}_{E}(\zeta(\mathcal{S}))=\left|\mathbb{K}_{1}\right|+\left|\mathbb{K}_{2}\right|-\operatorname{gr}(\zeta(\mathcal{S}))$.

Proof. Given that $\mathcal{S}=\mathbb{K}_{1} \times \mathbb{K}_{2}$ is a finite commutative ring, then each vertex of the form $(u, 0)$ of the zero-divisor graph $\zeta(\mathcal{S})$ is adjacent to each $(0, v)$ and vice versa. So, the vertex set of $\zeta(\delta)$ can be partitioned into two disjoint sets, say $U=$ $\left\{(u, 0): u \in \mathbb{K}_{1}^{*}\right\} \quad$ and $V=\left\{(0, v): v \in \mathbb{K}_{2}^{*}\right\}$. Hence, $\zeta(\mathcal{S}) \cong K_{m-1, n-1}$ and $\operatorname{gr}(\zeta(\mathcal{S}))=4$ which implies by Theorem 2 that $\operatorname{dim}_{E}(\zeta(\mathcal{S}))=\left|\mathbb{K}_{1}\right|+\left|\mathbb{K}_{2}\right|-\operatorname{gr}(\zeta(\mathcal{S}))$.

We know that we can break down any positive integer $m$ into set of prime numbers, resulting in the original number after multiplying. We are interested in finding the edge metric dimension of $\zeta\left(\mathbb{Z}_{m}\right)$ when $n=2 p$ and $n=p q$ where $p$ and $q$ are distinct primes. In both cases, the zero-divisor graph is the complete bipartite graph. The zero divisors of $\mathbb{Z}_{m}$ when $n=p q$ can be partitioned into two disjoint sets by taking all the multiples of $p$ in one set and the multiples of $q$ in the other set.

Corollary 2. Let $\mathcal{S}$ be a finite commutative ring with unity and $\mathcal{S} \cong \zeta\left(\mathbb{Z}_{m}\right)$, where $n=p q$ and $p$, $q$ are distinct primes $(p>2)$. Then, $\operatorname{dim}_{E}\left(\zeta\left(\mathbb{Z}_{m}\right)\right)=|\mathscr{Z}(\mathcal{S})|-2$.

Proposition 3. Let $\zeta(\mathcal{S})$ be a graph associated to a finite commutative ring $\mathcal{S}$. Then, $\operatorname{dim}_{E}(\zeta(\mathcal{S}))=|\mathscr{Z}(\mathcal{S})|-1$ if $\mathscr{Z}(\mathcal{S})$ is an annihilator ideal.

Proof. Given that $\zeta(\delta)$ is a graph associated to a commutative ring $\mathcal{S}$ and $\mathscr{Z}(\mathcal{S})$ is an annihilator ideal, then by definition for any $r \in \mathscr{Z}(\mathcal{S})$, we have $r s=0$ for all $s \in \mathscr{Z}(\mathcal{S})$, which implies that $\zeta(\mathcal{S})$ is a complete graph. Hence, by Theorem $1, \operatorname{dim}_{E}(\zeta(\mathcal{S}))=|\mathscr{Z}(\mathcal{S})|-1$.

Proposition 4. If $\mathcal{S}$ is a finite local ring with maximal ideal $\mu$ and $\mu^{2}=\{0\}$, then $\operatorname{dim}_{E}(\zeta(\mathcal{S}))=|\zeta(\mathcal{S})|-1$.

Proof. Given that $\mathcal{S}$ is a finite local ring, $\mathfrak{S}(\mathcal{S})=\mathscr{Z}(\mathcal{S})$ and $\mathscr{Z}(\mathcal{S})=\mu$, where $\mathfrak{I}(\mathcal{S})$ is the Jacobian radical of $\mathcal{S}$ which is 


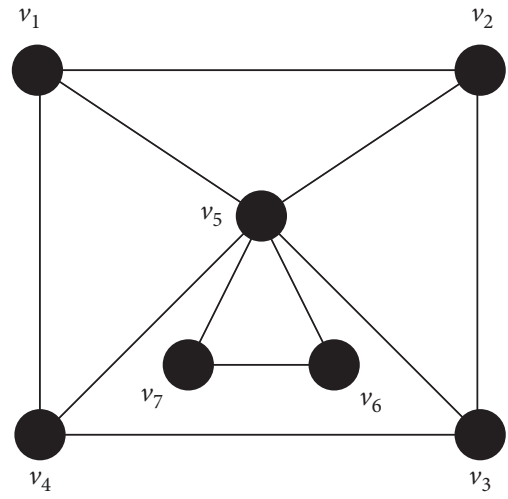

(a)

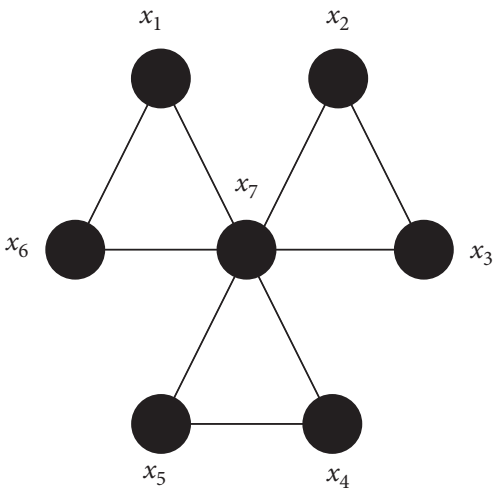

(b)

Figure 2: Zero-divisor graphs of polynomial rings.

the intersection of maximal ideals of $\mathcal{S}$. Thus, $\mathscr{Z}(\mathcal{S})$ is a nilpotent ideal and $\mathcal{S}$ is not a field which implies that $\operatorname{ann}(\mathscr{Z}(\mathcal{S})) \neq\{0\}$. So, ann $(\mathscr{Z}(\mathcal{S}))=\mathscr{Z}(\mathcal{S})$ since $\mu^{2}=\{0\}$, and therefore, $\zeta(\mathcal{S})$ is a complete graph. Hence, by Theorem $1, \operatorname{dim}_{E}(\zeta(\mathcal{S}))=|\zeta(\mathcal{S})|-1$.

Proposition 5. Let $\mathcal{S}$ be a reduced and $\mathscr{P}_{1}, \mathscr{P}_{2}$ be two prime ideals such that $\mathscr{P}_{1} \cap \mathscr{P}_{2}=\{0\}$. Then, $\operatorname{dim}_{E}(\zeta(\mathcal{S}))=\left|\mathscr{P}_{1}\right|+$ $\left|\mathscr{P}_{2}\right|-2 \omega(\zeta(\mathcal{S}))$.

Proof. First, we aim to prove that $\mathscr{Z}(\mathcal{S})=\mathscr{P}_{1} \cup \mathscr{P}_{2}$. Suppose that $r \in \mathscr{Z}(\mathcal{S}) / \mathscr{P}_{1} \cup \mathscr{P}_{2}$, so there exists a non-zero $s \in \mathcal{S}$ such that $r . s=0 \in \mathscr{P}_{1} \cap \mathscr{P}_{2}$. So, $s \in \mathscr{P}_{1} \cap \mathscr{P}_{2}$, which is a contradiction because $\mathscr{P}_{1} \cap \mathscr{P}_{2}=\{0\}$ as given. Also, $\mathscr{P}_{1} \cap \mathscr{P}_{2} \subseteq \mathscr{Z}(\mathcal{S})$, and hence $\mathscr{Z}(\mathcal{S})=\mathscr{P}_{1} \cup \mathscr{P}_{2}$. Now, we claim that $\zeta(\mathcal{S})$ is a complete bipartite graph with partite sets $V_{1}=\mathscr{P}_{1} /\{0\}$ and $V_{2}=\mathscr{P}_{2} /\{0\}$. Let $a, b \in V_{1}$ with $a b=0$. Then, $a b \in \mathscr{P}_{2}$, and therefore, either $a$ or $b \in V_{2}$, a contradiction. Thus, $\zeta(\mathcal{S})$ is a bipartite graph. Now, to show that $\zeta(\mathcal{S})$ is a complete bipartite graph, we take $a \in V_{1}$ and $b \in V_{2}$. So, $a b \in \mathscr{P}_{1}$ and $a b \in \mathscr{P}_{2}$; since both $\mathscr{P}_{1}$ and $\mathscr{P}_{2}$ are ideals, then $a b \in \mathscr{P}_{1} \cap \mathscr{P}_{2}=\{0\}$ which implies that $a b=0$ and so $\zeta(\delta)$ is a complete bipartite graph. Then, $\omega(\zeta(\mathcal{S}))=2$. Hence, by Theorem 2, $\operatorname{dim}_{E}(\zeta(\mathcal{S}))=\left|\mathscr{P}_{1}\right|+\left|\mathscr{P}_{2}\right|-2 \omega(\zeta(\mathcal{S}))$.

Theorem 8. Let $\mathcal{S}$ be a finite commutative ring with unity and $\mathcal{S} \cong \mathbb{Z}_{p}[r] /\left(r^{2}\right)$ or $\mathcal{S} \cong \mathbb{Z}_{p^{2}}$, where $p$ is a prime. Then, $\operatorname{dim}_{E}(\zeta(\delta))=p-2$.

Proof. Suppose that $\mathcal{S} \cong \mathbb{Z}_{p}[r] /\left(r^{2}\right)$; then, the elements of $\mathcal{S}$ are of the form $\left\{a r+b: a, b \in \mathbb{Z}_{p}\right\}$, and so the set of zero divisors of $\mathcal{S}$ is $\mathscr{Z}(\mathcal{S})=\{b r: b \in\{1, \ldots, p-1\}\}$ such that $r_{1} r \cdot r_{2} r=0$ for each $r_{1}, r_{2} \in\{1,2, \ldots, p-1\}$. Hence, $\zeta(\mathcal{S}) \cong K_{p-1}$.

Now, if $\mathcal{S} \cong \mathbb{Z}_{p^{2}}$, then its set of non-zero zero divisors is $\mathscr{Z}(\mathcal{S})=\{r p: r \in\{1, \ldots, p-1\}\}$ such that $r_{1} p \cdot r_{2} p=0$ for each $r_{1}, r_{2} \in\{1,2, \ldots, p-1\}$. Observe that $\zeta(\mathcal{S}) \cong K_{p-1}$, and so by Theorem $1, \operatorname{dim}_{E}(\zeta(\mathcal{S}))=p-2$.

Let us now determine the edge metric dimension of the zero-divisor graph of the ring of Gaussian integers $\zeta\left(\mathbb{Z}_{m}[i]\right)$.
As stated above, the set of Gaussian integers is of the form $\left\{r+i s: r, s \in \mathbb{Z}\right.$ and $\left.i^{2}=-1\right\}$ and the set of Gaussian integers modulo $m$ is of the form $\mathbb{Z}_{m}[i]=\left\{r+i s: r, s \in \mathbb{Z}_{m}\right\}$. A Gaussian prime is the prime element in $\mathbb{Z}[i]$ and the Gaussian primes can be described as

(1) $1+i$ and $1-i$ are Gaussian primes.

(2) If $p$ is a prime integer with $p \equiv 1(\bmod 4)$ and $p=$ $a^{2}+b^{2}$ for some integers $a$ and $b$, then $a+i b$ and $a-i b$ are Gaussian primes.

(3) If $q$ is a prime integer with $q \equiv 3(\bmod 4)$, then $q$ is a Gaussian prime.

Furthermore, if $q$ is a Gaussian prime, then its complex conjugate and its associates $-q$, $i q$, and $-i q$ are also Gaussian primes. If $n=p \equiv 3(\bmod 4)$, then $\operatorname{dim}_{E}\left(\zeta\left(\mathbb{Z}_{m}[i]\right)\right)$ is undefined; since $\mathbb{Z}_{m}[i]$ is a field, $\zeta\left(\mathbb{Z}_{m}[i]\right)$ is an empty graph.

Theorem 9. Let $\mathcal{S}$ be a finite commutative ring with unity and $\mathcal{S} \cong \zeta\left(\mathbb{Z}_{m}[i]\right)$. Then,

(1) For $n=p^{2}, \operatorname{dim}_{E}\left(\zeta\left(\mathbb{Z}_{m}[i]\right)\right)=p^{2}-2$.

(2) For $p_{j} \equiv 3(\bmod 4), \quad j=1,2 \quad \operatorname{dim}_{E}\left(\zeta\left(\mathbb{Z}_{m}[i]\right)\right)=$ $p_{1}^{2}+p_{2}^{2}-2 \omega \zeta\left(\mathbb{Z}_{p_{1}, p_{2}}[i]\right)$.

(3) For $n=p \equiv 1(\bmod 4)$ with $p=a^{2}+b^{2}, \operatorname{dim}_{E}\left(\zeta\left(\mathbb{Z}_{m}\right.\right.$ $[i]))=2 p-\operatorname{gr}\left(\zeta\left(\mathbb{Z}_{m}[i]\right)\right)$.

Proof

(1) It was shown in Theorem 15 [17] that $n=p^{2}\left(\zeta\left(\mathbb{Z}_{m}[i]\right)\right)$ is a complete graph, and by Theorem $1, \operatorname{dim}_{E}\left(\zeta\left(\mathbb{Z}_{m}[i]\right)\right)=p^{2}-2$.

(2) Given that $p_{1}, p_{2}$ are primes such that $p_{j} \equiv 3(\bmod 4)$, for $j=1,2$, then $\zeta\left(\mathbb{Z}_{p_{1}, p_{2}}[i]\right)$ is a complete bipartite graph, since $\mathbb{Z}_{p_{1}, p_{2}}[i] \stackrel{p_{1}, \mathbb{Z}_{p_{1}}}{\cong}[i] \times$ $\mathbb{Z}_{p_{2}}[i]$ as a direct product of two fields. Also, for a complete bipartite graph $G$, the clique number $\omega(G)=2$, and hence by Theorem 2, $\operatorname{dim}_{E}\left(\zeta\left(\mathbb{Z}_{p_{1}, p_{2}}[i]\right)\right)=p_{1}^{2}+p_{2}^{2}-2 \omega \zeta\left(\mathbb{Z}_{p_{1}, p_{2}}[i]\right)$.

(3) If $p \equiv 1(\bmod 4)$ with $p=a^{2}+b^{2}$, then $\zeta\left(\mathbb{Z}_{p}[i]\right)$ is a complete bipartite graph $K_{p-1, p-1}$ with partite sets 
TABle 1: Rings with same metric dimension, edge metric dimension, and upper dimension.

\begin{tabular}{l}
\hline Commutative rings $\operatorname{dim}\left((\zeta(\mathcal{S}))=\operatorname{dim}_{E}(\zeta(\mathcal{S}))=\operatorname{dim}^{+}(\zeta(\mathcal{S}))\right.$ \\
$\mathcal{S}$ \\
$\mathbb{Z}_{6}, \mathbb{Z}_{8}, \mathbb{Z}_{9}, \mathbb{Z}_{2} \times \mathbb{Z}_{2}$, \\
$\mathbb{Z}_{3}[r] /\left(r^{2}\right)$, \\
$\mathbb{Z}_{2}[r] /\left(r^{3}\right)$, \\
$\mathbb{Z}_{4}[r] /\left(2 r, r^{2}-2\right)$ \\
$\mathbb{Z}_{3} \times \mathbb{Z}_{3}$, \\
$\mathbb{F}_{4}[r] /\left(r^{2}\right)$, \\
$\mathbb{Z}_{4}[r] /\left(r^{2}+r+1\right)$, \\
$\mathbb{Z}_{4}[r] /(2, r)^{2}$, \\
$\mathbb{Z}_{2}[r, s] /(r, s)^{2}$ \\
$\mathbb{K}_{1} \times \mathbb{K}_{2}$, both $\mathbb{K}_{1}$ and \\
$\mathbb{K}_{2}$ \\
are finite fields of \\
order \\
greater or equal to 3 \\
$\mathbb{Z}_{p}[r] /\left(r^{2}\right)$ or $\mathbb{Z}_{p^{2},}$ \\
where $p$ is a prime \\
\hline
\end{tabular}

$\langle a+i b\rangle /\{0\} \quad$ and $\langle a-i b\rangle /\{0\}, \quad$ since $\mathbb{Z}_{p}[i] \cong \mathbb{Z}[i] /\langle p\rangle \cong \mathbb{Z}[i] /\langle a+i b\rangle \times \mathbb{Z}[i] /\langle a-i b\rangle$. Also, for a complete bipartite graph $G$, then girth $\operatorname{gr}(G)=2$, and hence by Theorem 2, $\operatorname{dim}_{E}\left(\zeta\left(\mathbb{Z}_{m}[i]\right)\right)=2 p-\operatorname{gr}\left(\zeta\left(\mathbb{Z}_{m}[i]\right)\right)$

Remark 1. We know that 2 is not a Gaussian prime, since $2=(1-i)(1+i)$; however, $2=-i(1+i)^{2}$, so $\mathbb{Z}_{2}[i]$ is isomorphic to the local ring $\mathbb{Z}[i] /\left\langle(1+i)^{2}\right\rangle$ having unique maximal ideal $\{0,1+i\}$. Thus, we have $V\left(\zeta\left(\mathbb{Z}_{2}[i]\right)\right)=\{1+i\}$, which implies that $\zeta\left(\mathbb{Z}_{2}[i]\right)$ is a graph with one vertex and no edges. Hence, $\operatorname{dim}_{E}\left(\zeta\left(\mathbb{Z}_{2}[i]\right)\right)=0$.

Now, we prove the bounds for edge metric dimension of zero-divisor graph associated to rings. Moreover, the following result gives a relation between the maximum degree, edge metric dimension of $\zeta(\mathcal{S})$, and the diameter.

Theorem 10. Let $\mathcal{S}$ be a finite commutative ring with unity and let $\zeta(\mathcal{S})$ be its zero-divisor graph such that $|\mathscr{Z}(\mathcal{S})| \geq 5$. Then,

$$
\left\lceil\log _{2}(\rho)\right\rceil \leq \operatorname{dim}_{E}(\zeta(\mathcal{S})) \leq|\mathscr{Z}(\mathcal{S})|-\delta
$$

where $\rho$ is the maximum degree and $\delta$ is the diameter of $\zeta(\mathcal{S})$.

Proof. First, we prove that $\left[\log _{2}(\rho)\right\rceil \leq \operatorname{dim}_{E}(\zeta(\delta))$. Let $S=$ $\left\{v_{1}, v_{2}, \ldots, v_{r}\right\}$ be the minimum edge resolving set for $\zeta(\delta)$. Let $u \in V(\zeta(\mathcal{S}))$ with $\operatorname{deg}(u)=\rho$ and $\left\{e_{1}, e_{2}, \ldots, e_{\rho}\right\}$ be the set of all edges incident to $u$. For each $i \in\{1,2, \ldots, r\}$, let $d_{i}=\min \left\{d(u, v)\right.$ : visincidentto $\left.v_{i}\right\}$. Then, $d\left(v_{i}, e_{j}\right)$ is $d_{i}$ or $d_{i}+1$. Therefore, the size $\mathscr{D}=\left\{D\left(e_{j} \mid S\right): j=1, \ldots, \rho\right\}$ is at most $2^{r}$. Since $D\left(e_{j} \mid S\right) \neq D\left(e_{k} \mid S\right)$ for $j \neq k, \rho(G) \leq 2^{r}$, and the assertion follows.

Now, to show that $\operatorname{dim}_{E}(\zeta(\mathcal{S})) \leq|\mathscr{Z}(\mathcal{S})|-\delta$, suppose that for $u, v \in V(\zeta(\delta)), \quad d(u, v)=\sup : \quad\{d(x, y) \cdot x, y$ $\in \mathscr{Z}(\mathcal{S})\}=\delta$, i.e., $d(u, v)$ is the diameter of $\zeta(\mathcal{S})$ and let $u=v_{o}, v_{1}, \ldots, v_{\delta}=v$ be $u-v$ path of length $\delta$, where $v_{o} \cdot v_{1}=e_{1}, \quad v_{1} \cdot v_{2}=e_{2}, \quad \ldots, \quad v_{\delta-1} \cdot v_{\delta}=e_{\delta}$. Suppose
$S=V(\zeta(\mathcal{S})) /\left\{v_{1}, v_{2}, \ldots, v_{\delta}\right\}$, and since $u \in S$ and $d\left(u, e_{i}\right)=$ $i-1$ for $i=\{1, \ldots, \delta\}$, it follows that $S$ is an edge metric generator for $\zeta(\mathcal{S})$ of cardinality $|\mathscr{Z}(\mathcal{S})|-\delta$. Hence, $\operatorname{dim}_{E}(\zeta(\delta)) \leq|\mathscr{Z}(\delta)|-\delta$.

When are the metric dimension, edge metric dimension, and upper dimension the same?

In the previous section, bounds for edge metric dimension of zero-divisor graphs were given. In this section, we provide the commutative rings for which the zero-divisor graph $\zeta(\mathcal{S})$ has same metric dimension $\operatorname{dim}_{E}(\zeta(\delta)$ ), edge metric dimension $\operatorname{dim}_{E}(\zeta(\mathcal{S})$ ), and upper dimension $\operatorname{dim}^{+}(\zeta(\delta))$ (see Table 1$)$.

\section{Conclusion}

In this paper, we discussed the bounds for the edge metric dimension of graphs associated to commutative rings for some rings such as ring of integers modulo $m$, polynomial rings, and the ring of Gaussian integers modulo $m$. Finally, we provide the general bound for edge metric dimension of zero-divisor graphs in terms of maximum degree of $\zeta(\mathcal{S})$ and its diameter $\delta$ [21].

\section{Data Availability}

The data used to support the findings of this study are included within the article.

\section{Conflicts of Interest}

The authors declare that they have no conflicts of interest.

\section{References}

[1] I. Beck, "Coloring of commutative rings," Journal of Algebra, vol. 116, no. 1, pp. 208-226, 1988.

[2] D. D. Anderson and M. Naseer, "Beck's coloring of a commutative ring," Journal of Algebra, vol. 159, no. 2, pp. 500-514, 1993.

[3] D. F. Anderson and P. Livingston, "The zero divisor graph of a commutative ring," Journal of Algebra, vol. 217, no. 2, pp. 434-447, 1999.

[4] S. Redmond, "The zero-divisor graph of a non-commutative ring," International Journal of Commutative Rings, vol. 1, no. 4, pp. 203-211, 2002.

[5] S. P. Redmond, "An ideal-based zero-divisor graph of a commutative ring," Communications in Algebra, vol. 31, no. 9, pp. 4425-4443, 2003.

[6] N. Ashrafi, H. R. Maimani, M. R. Pournaki, and S. Yassemi, "Unit graphs associated with rings," Communications in Algebra, vol. 38, no. 8, pp. 2851-2871, 2010.

[7] S. Spiroff and C. Wickham, "A zero divisor graph determined by equivalence classes of zero divisors," Communications in Algebra, vol. 39, no. 7, pp. 2338-2348, 2011.

[8] D. F. Anderson and A. Badawi, "The total graph of a commutative ring," Journal of Algebra, vol. 320, no. 7, pp. 2706-2719, 2008.

[9] A. Azimi, A. Erfanian, and M. Farrokhi, "The Jacobson graph of commutative rings," Journal of Algebra and Its Applications, vol. 12, no. 3, Article ID 1250179, 2012.

[10] S. Pirzada, An Introduction to Graph Theory, University Press, Orient Black- swan, Hyderabad India, 2012. 
[11] D. B. West, Introduction Graph Theory, Prentice-Hall, Hoboken, NJ, USA, 2001.

[12] I. Kaplansky, Commutative Rings, University of Chicago Press, Chicago, IL, USA, 1974.

[13] M. F. Atiyah and I. G. MacDonald, Introduction to Commutative Algebra, Westview Press, Boulder, CO, USA, 1969.

[14] S. P. Redmond, "On zero divisor graphs of small finite commutative rings," Discrete Mathematics, vol. 307, no. 9-10, pp. 1155-1166, 2007.

[15] S. Redmond and S. Szabo, "When metric and upper dimensions differ in zero divisor graphs of commutative rings," Discrete Mathematics Letters, vol. 5, pp. 34-40, 2021.

[16] A. Ali, "A survey of antiregular graphs," Contributions to Mathematics, vol. 1, pp. 67-79, 2020.

[17] E. A. Osba, S. Al-Addasi, and N. A. Jaradeh, "Zero divisor graph for the ring of Gaussian integers modulo n," Communications in Algebra, vol. 36, no. 10, pp. 3865-3877, 2008.

[18] A. Kelenc, N. Tratnik, and I. G. Yero, "Uniquely identifying the edges of a graph: the edge metric dimension," Discrete Applied Mathematics, vol. 251, pp. 204-220, 2018.

[19] S. Pirzada, M. Aijaz, and S. P. Redmond, "Upper dimension and bases of zero-divisor graphs of commutative rings," AKCE International Journal of Graphs and Combinatorics, vol. 17, no. 1, pp. 168-173, 2019.

[20] S. P. Redmond, "Cut vertices and degree one vertices of zerodivisor graphs," Communications in Algebra, vol. 40, no. 8, pp. 2749-2756, 2012.

[21] D. F. Anderson, A. Frazier, A. Lauve, and P. S. Livingston, The Zero-Divisor Graph of A Commutative Ring Ii, Marcel Dekker, New York, NY, USA, 2001. 